\title{
Computed tomography depiction of normal inguinal lymph nodes in children
}

\author{
M.S. Dogan1, G. Koc², S. Doganay³, S. Dogan³, F. Elmalı4, S. Ciraci³ , S.B. Gorkem³, \\ M. Guzel ${ }^{5}$, A. Coskun ${ }^{3}$ \\ 'Department of Radiology, School of Medicine, Istanbul Medipol University, Istanbul, Turkey \\ ${ }^{2}$ Department of Paediatric Radiology, School of Medicine, Ege University, Izmir, Turkey \\ ${ }^{3}$ Department of Paediatric Radiology, School of Medicine, Erciyes University, Kayseri, Turkey \\ ${ }^{4}$ Department of Bioistatistics, School of Medicine, Katip Celebi University, Izmir, Turkey \\ ${ }^{5}$ Department of Paediatric Surgery, School of Medicine, Erciyes University, Kayseri, Turkey
}

[Received: 17 November 2019; Accepted: 20 December 2019]

Background: The aim of the study was to establish computed tomography (CT) characteristics, distribution and provide normative data about size of normal inguinal lymph nodes in a paediatric population.

Materials and methods: Four hundred eighty-one otherwise healthy children (147 girls, mean age: 8.87, range 0-17 years) underwent pelvic CT in the setting of high-energy trauma were included in the study. Both axial and coronal 1.25- $\mathrm{mm}$ reconstructions were evaluated for the presence, location (deep or superficial), number, presence of fat attenuation, and shape of the lymph nodes, short-axis diameter of the biggest lymph node for each of right and left inguinal regions.

Results: A total of 7556 lymph nodes were detected in 481 subjects (the mean count of superficial and deep inguinal lymph nodes was 13.35 [range 6-23] and 2.36 [range 0-7] per subject, respectively): 15\% (1135/7556) deep located, 85\% (6421/7556) superficially located, 86.6\% (6547/7556) with fat attenuation, 99.2\% (7496/7556) oval in shape, 0.8\% (60/7556) spherical. The short-axis diameter of the lymph nodes increased with age. Pearson's correlation coefficient for superficial and deep lymph nodes in boys and girls, respectively: 0.538 ( $p<0.001), 0.504$ $(p<0.001), 0.452(p<0.001)$ and 0.268 ( $p<0.001)$. The mean maximum short-axis diameters in different age groups and gender varied between $6.33 \pm$ $\pm 0.85 \mathrm{~mm}$ and $8.68 \pm 1.33 \mathrm{~mm}$ for superficial, $3.62 \pm 1.16 \mathrm{~mm}$ and $5.83 \pm$ $\pm 1.05 \mathrm{~mm}$ for deep inguinal lymph nodes.

Conclusions: Inguinal lymph nodes were multiple, commonly contained fat, and were oval in shape. The data determined about inguinal lymph node size in different paediatric age groups may be applicable as normative data in daily clinical CT evaluation practice. (Folia Morphol 2020; 79, 4: 799-804)

Key words: paediatric, inguinal, computed tomography, lymph node

\section{INTRODUCTION}

Lymphadenopathy is a commonly encountered clinical sign indicating either infectious or malignant pathologies in children. Imaging characteristics in- cluding being round than oval in shape, disappearance of fatty hilum, asymmetrical thickening of the cortex and enlargement have been reported to be associated with abnormal lymph nodes [3]. Inguinal

Address for correspondence: G. Koc, MD, Ege University, Faculty of Medicine, Department of Paediatric Radiology, İzmir, Turkey, 35090, tel: +90 505 4780123, e-mail: ggulkoc@gmail.com 
lymph nodes are usually identified incidentally in the field-of-view of pelvic or abdominopelvic computed tomography (CT) examinations. In order to differentiate normal lymph nodes from pathologic ones on $\mathrm{CT}$, to be aware of normal imaging characteristics and size limits is crucial.

There have been very few studies providing information about size range and imaging characteristics of normal inguinal lymph nodes in adult age group $[1,4,9,10]$. However, to our knowledge, there have been neither established size criteria nor a study assessing imaging characteristics and distribution of inguinal lymph nodes on CT in children.

Computed tomography is a frequently used imaging modality to evaluate patients suffered from high-energy trauma. Because of well-known harmful effects of exposure to the radiation, ethically it is impossible to obtain $\mathrm{CT}$ examinations with a healthy paediatric volunteer study group. However, we suggest that otherwise healthy children who underwent $\mathrm{CT}$ in the setting of high-energy trauma could be considered as a sample study group and may represent normal healthy children population. Therefore, in this study we aimed to establish CT characteristics, distribution and provide normative data about size of inguinal lymph nodes in children with high-energy trauma.

\section{MATERIALS AND METHODS}

The current study complied with the Declaration of Helsinki and was approved by the Ethics Committee of the institution. (Approval number: 2016/445, date: 29.07.2016).

\section{Subjects}

Due to ethical considerations of $\mathrm{CT}$ associated radiation exposure, a retrospective study was conducted. A search of radiology information system and medical records for the paediatric patients who underwent abdominopelvic or pelvic CT in the setting of high-energy trauma between July 2012 and December 2015 revealed 485 otherwise healthy children. Of these, 4 patients were excluded due to causes (motion artefacts in two, and subcutaneous emphysema in rest) that compromised the evaluation of inguinal lymph nodes. Therefore, 481 children (147 girls, mean age: 8.87 , range $0-17$ years) were recruited for the study.

\section{CT examination procedure}

The CT examinations were performed on a clinical CT scanner (GE Light Speed 16; General Electric Med- ical Systems, Waukesha, WI, USA) with $16 \times 0,625$ collimation. The exposure settings were adjusted to patient size and ranged from 40 to $340 \mathrm{mAs}$ and from 80 to $120 \mathrm{kV}$. A data matrix of $512 \times 512$ was chosen. Axial images with slice thickness of $1.25 \mathrm{~mm}$ were reconstructed including the abdominopelvic or pelvic area. $90.2 \%(434 / 481)$ of examinations were performed with $1 \mathrm{cc} / \mathrm{kg}$ intravenous iodinated contrast medium administration.

\section{CT image analysis}

All CT examinations were retrospectively evaluated by a paediatric radiologist with 6 years of post-residency experience (M. S. D). To accurately investigate inguinal lymph nodes, both axial images and coronal plane reformatted images were evaluated. According to the location, inguinal lymph nodes were grouped as superficial and deep. The former included lymph nodes located at anterior or lateral to the superficial femoral vessels and the saphenous veins at or inferior to the level of inguinal ligament in the subcutaneous tissue, whereas the latter consisted of lymph nodes located along the common femoral vessels, generally medial to the common femoral vein (Fig. 1) $[1,5,8]$. The inguinal ligament and the origins of the circumflex iliac and inferior epigastric vessels were regarded as landmarks to differentiate the deep inguinal lymph nodes from the medial chain of external iliac nodes [8]. Thus, inguinal lymph nodes were defined as superficial or deep for each of left and right sides and subsequently four inguinal areas (right and left superficial/deep) were described for each subject. The number of lymph nodes was counted for each area, and the presence of fat attenuation and shape (as spherical or oval) of the lymph node were noted for each of lymph nodes. The short axis diameter of the biggest inguinal lymph node for each of four areas was measured by using an electronic calliper tool on an available diagnostic screen.

\section{Statistical analysis}

Statistical analyses were carried out using SPSS v.22.0 (IBM Corp., Armonk, NY, USA). Data were expressed either as frequencies or mean \pm standard deviation (SD). Shapiro-Wilk's test was implemented, a histogram and q-q plot were examined to assess the data normality. Levene's test was used to assess the variance homogeneity. Paired samples t-test was applied to compare variables between deep and superficial location and left and right 


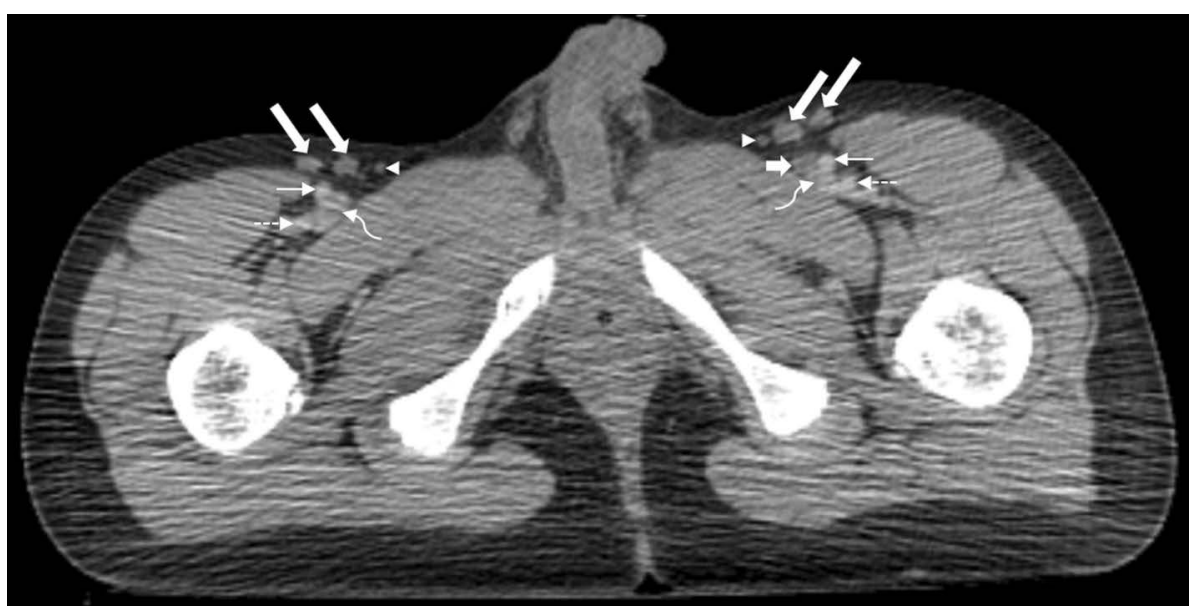

Figure 1. Pelvic computed tomography after contrast-medium injection in an 11-year-old boy revealed bilateral superficial inguinal lymph nodes (long thick arrows) and left deep inguinal lymph node (short thick arrow). All lymph nodes in this image had central hypodensity compatible with fat attenuation. (Thin arrows: superficial femoral arteries, dashed thin arrows: deep femoral arteries, curved arrows: common femoral veins, arrowheads: saphenous veins).

side. Student t-test was used to compare variables between males and females. Pearson's correlation analysis was implemented to determine the relationship between lymph node size and age groups. Scatter charts were drawn with \pm 2 SD values besides means. A p-value $<0.05$ was considered statistically significant.

\section{RESULTS}

A total of 7556 lymph nodes were detected in 481 subjects. Of these, $99.2 \%(n=7496)$ were oval in shape, whereas $0.8 \%(n=60)$ were spherical. There were a total of 6421 ( $85 \%, 3209$ on right side) superficial and 1135 (15\%, 584 on right side) deep inguinal lymph nodes. The mean count of superficial and deep inguinal lymph nodes was $13.35 \pm 3.27$ (range 6-23) and $2.36 \pm 1.46$ (range $0-7$ ) per subject, respectively. In $12.9 \%(n=62)$ of the subjects, no deep inguinal lymph nodes were detected in neither side. With regard to number of superficial and deep lymph nodes, there was no statistical difference between left versus right side $(p=0.994, p=0.109)$ or between male versus female ( $p=0.099, p=0.505$, respectively). However, there was statistically significant difference in number of lymph nodes between superficial and deep regions $(p<0.001)$.

Fat attenuation was detected in $86.6 \%(n=6547$, $73.9 \%$ [839/1135] of deep, 88.9\% [5708/6421] of superficial) of all lymph nodes. The presence of fat attenuation was significantly more common on superficial lymph nodes compared to deep ones $(p<0.001)$.
The overall mean short axis diameter of the biggest lymph node (mean maximum short axis diameter) of superficial lymph nodes (mean $7.45 \pm 1.59$ $\mathrm{mm}$, range 3.6-15.4 $\mathrm{mm}$ ) was significantly higher ( $p<0.001$ ) compared to deep lymph nodes (mean $4.68 \pm 1.38 \mathrm{~mm}$, range $1.3-10.2 \mathrm{~mm} ; \mathrm{p}<0.001$ ). In point of short axis diameters of superficial and deep lymph nodes, there was no statistical difference between left versus right side $(p=0.739, p=0.121$, respectively) whereas there were statistical differences between male versus female $(p=0.044, p=0.010$, respectively). The mean maximum short axis diameters of the biggest lymph node for superficial and deep lymph nodes were $7.55 \pm 1.67 \mathrm{~mm}$ (range 3.7 $-15.4 \mathrm{~mm}$ ) and $4.79 \pm 1.42 \mathrm{~mm}$ (range 1.3-10.2 mm) in boys and $7.23 \pm 1.39 \mathrm{~mm}$ (range $3.6-11.5 \mathrm{~mm}$ ) and $4.41 \pm 1.27 \mathrm{~mm}$ (range 1.5-8 mm) in girls.

The short-axis diameters of the lymph nodes were detected to increase with age. Pearson's correlation coefficient for superficial and deep lymph nodes in boys and girls was: $0.538(p<0.001), 0.504(p<0.001)$, $0.452(p<0.001)$ and $0.268(p<0.001)$, respectively. According to the different age groups, the mean maximum short-axis size of lymph nodes ranged from $6.33 \pm 0.85$ to $8.68 \pm 1.33 \mathrm{~mm}$ and $3.92 \pm 0.84$ to $5.83 \pm 1.05 \mathrm{~mm}$ for superficial and deep lymph nodes in boys respectively. In girls, it ranged from $6.34 \pm 1.55$ to $8.51 \pm 1.43 \mathrm{~mm}$ and $3.62 \pm 1.16$ to $5.16 \pm 1.44 \mathrm{~mm}$ for superficial and deep lymph nodes, respectively. For superficial and deep lymph nodes in boys and girls age-related maximum values at $2 \mathrm{SD}$ above the mean of $11.34,9.1,11.37$ and $8.04 \mathrm{~mm}$ 
Table 1. The mean maximum short-axis diameter of superficial and deep inguinal lymph nodes in girls

\begin{tabular}{lcccc}
\hline Age groups [year] & Subjects $(\mathbf{n}=\mathbf{1 4 7})$ & Diameter for superficial lymph nodes* & Subjects $(\mathbf{n}=\mathbf{1 2 8})^{\text {Diameter for deep lymph nodes* }}$ \\
\hline $0-1$ & 20 & $6.34 \pm 1.55$ & 17 & $3.62 \pm 1.16$ \\
$2-3$ & 23 & $6.92 \pm 1.12$ & 18 & $4.66 \pm 1.35$ \\
$4-5$ & 18 & $6.92 \pm 1.28$ & 17 & $4.39 \pm 1.16$ \\
$6-7$ & 14 & $6.22 \pm 0.84$ & 12 & $3.65 \pm 0.7$ \\
$8-9$ & 14 & $7.02 \pm 0.75$ & 11 & $4.06 \pm 1.06$ \\
$10-11$ & 7 & $6.68 \pm 1.46$ & 7 & $3.76 \pm 0.95$ \\
$12-13$ & 14 & $8.51 \pm 1.43$ & 13 & $5.16 \pm 1.44$ \\
$14-15$ & 20 & $8.23 \pm 0.91$ & 16 & $5.04 \pm 1.25$ \\
$16-17$ & 17 & $7.99 \pm 1.07$ & 17 & $4.85 \pm 1.14$ \\
\hline
\end{tabular}

*Lymph node sizes were expressed as mean \pm standard deviation in millimetres.

Table 2. The mean maximum short-axis diameter of superficial and deep inguinal lymph nodes in boys

\begin{tabular}{lcccc}
\hline Age groups [year] & Subjects $(\mathbf{n}=\mathbf{3 3 4})$ & Diameter for superficial lymph nodes* & Subjects $(\mathbf{n}=\mathbf{2 9 1})$ & Diameter for deep lymph nodes* $^{*}$ \\
\hline $0-1$ & 28 & $6.33 \pm 0.85$ & 22 & $3.92 \pm 0.84$ \\
$2-3$ & 32 & $6.44 \pm 0.98$ & 32 & $3.93 \pm 1.08$ \\
$4-5$ & 34 & $6.62 \pm 1.22$ & 30 & $4.02 \pm 0.63$ \\
$6-7$ & 37 & $6.81 \pm 1.29$ & 33 & $4.24 \pm 1.05$ \\
$8-9$ & 29 & $6.91 \pm 1.42$ & 22 & $4.48 \pm 0.87$ \\
$10-11$ & 40 & $7.89 \pm 1.64$ & 35 & $4.72 \pm 1.27$ \\
$12-13$ & 39 & $8.27 \pm 1.31$ & 34 & $5.28 \pm 1.48$ \\
$14-15$ & 60 & $8.61 \pm 1.85$ & 55 & $5.72 \pm 1.69$ \\
$16-17$ & 35 & $8.68 \pm 1.33$ & 28 & $5.83 \pm 1.05$ \\
\hline
\end{tabular}

*Lymph node sizes were expressed as mean \pm standard deviation in millimetres.

were calculated. Detailed results are revealed in Tables 1 and 2. Graphic illustrations of the mean maximum short axis diameters of the superficial and deep lymph nodes for both boys and girls according to age groups are shown in Figures 2-5.

\section{DISCUSSION}

The lymph nodes in either superficial or deep location are encountered on almost every pelvic CT examinations in children. In our opinion, the most striking result of this study is that it has provided information about normal limits of short-axis diameters per location and age for children aged 0-17 years. We have founded short axis diameter values at 2 SD above the mean, increasing with age up to $11.34 \mathrm{~mm}$, $9.1 \mathrm{~mm}, 11.37 \mathrm{~mm}$, and $8.04 \mathrm{~mm}$ for superficial and deep lymph nodes in boys and girls, respectively. In the clinical approach, while a maximum diameter of $10 \mathrm{~mm}$ is being considered as normal for lymph nodes

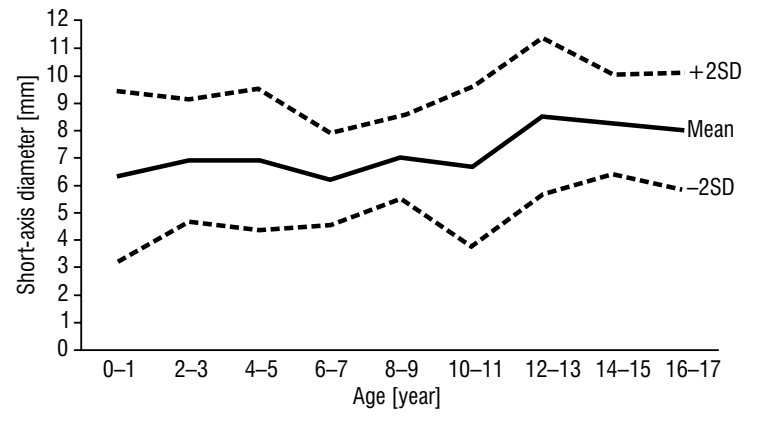

Figure 2. The mean maximum short-axis diameter of superficial lymph nodes plotted against age for girls. The $x$-axis represents age in years and the $y$-axis represents the short-axis diameter in millimetres; SD — standard deviation.

in general, some authors have suggested a maximum diameter of $15 \mathrm{~mm}$ for inguinal lymph nodes as normal in children $[6,7]$. However, these criteria are used in the clinical practice and do not depend on any imaging method. In a limited number of studies 


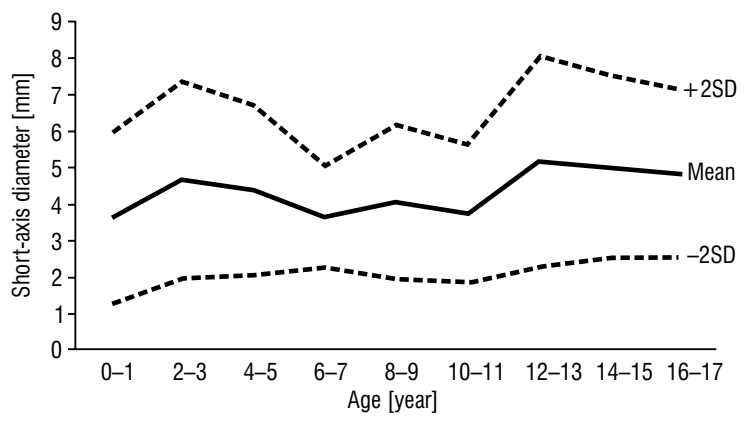

Figure 3. The mean maximum short-axis diameter of deep lymph nodes plotted against age for girls. The $x$-axis represents age in years and the $y$-axis represents the short-axis diameter in millimetres; SD standard deviation.

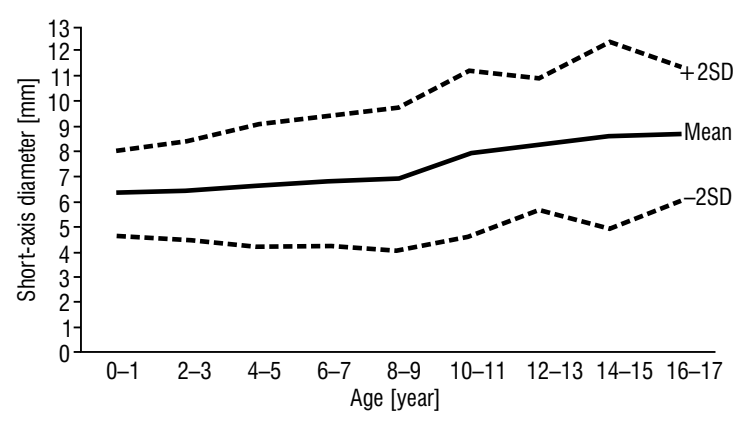

Figure 4. The mean maximum short-axis diameter of superficial lymph nodes plotted against age for boys. The $x$-axis represents age in years and the $y$-axis represents the short-axis diameter in millimetres; SD — standard deviation.

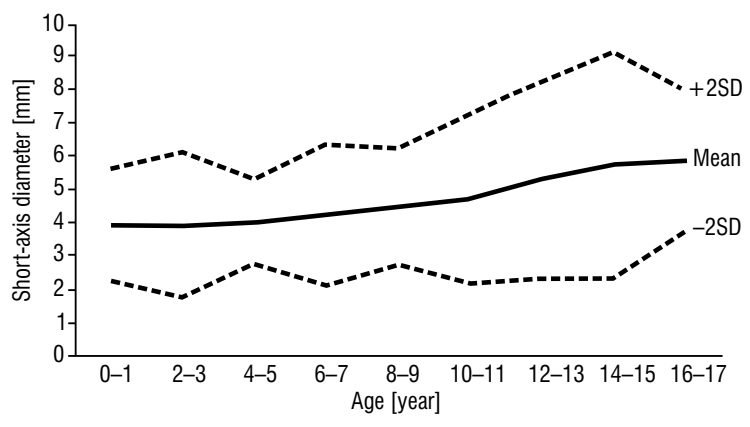

Figure 5. The mean maximum short-axis size of deep lymph nodes plotted against age for boys. The $x$-axis represents age in years and the $y$-axis represents the short-axis diameter in millimetres; SD - standard deviation.

assessing size of normal inguinal lymph nodes on different imaging modalities in adults, upper limit of short axis diameter of inguinal lymph nodes varies between 7 and $15 \mathrm{~mm}[1,4,9,10]$. The study evaluated inguinal lymph nodes in asymptomatic patients on CT revealed a mean short axis of $5.4 \mathrm{~mm}$, and a value of $8.8 \mathrm{~mm}$ at $2 \mathrm{SD}$ above the mean for all inguinal lymph nodes [1]. To our knowledge, there has been no study assessing normal inguinal lymph nodes on CT in children. We also aimed to determine lymph node sizes separately for superficial and deep location according to gender.

Furthermore, this study revealed the positive correlation of short-axis diameter of inguinal lymph nodes and age for both superficial and deep located lymph nodes in boys and girls. However, the positive correlation was found to be stronger in boys than girls. The mean maximum short axis diameter-age curves in boys were smoother compared to that found in girls (Figs. 2-5). All these differences may be attributed to relatively large number of boys compared to that of girls to represent more accurately normal population.

Our study has also established CT characteristics and distribution of normal inguinal lymph nodes in children. Inguinal lymph nodes were multiple and symmetrical in size and number, commonly contained fat, and oval in shape similar to the CT study in adults [1]. The mean count of superficial lymph nodes was 13.35 (range 6-23), and the mean count of deep inguinal lymph nodes was 2.36 (range $0-7$ ) per subject. These data are similar to the results of a cadaveric dissection study [2] in which the mean numbers of superficial and deep lymph nodes were observed as 13.60 and 1.71 , respectively. Our result of mean count of superficial lymph nodes also agree with the CT study [1] in which the mean number of superficial lymph nodes was detected as 11.7, whereas the mean number of deep lymph nodes (1.2 per patient) in that study differs from that of ours.

This study showed that there was a significant difference between superficial and deep lymph nodes in terms of number and size in favour of superficial, consistent with the literature [1, 2]. The ratio of presence of fat attenuation in superficial lymph nodes (88.9\%) was significantly higher than in deep lymph nodes (73.9\%) as in the literature [1]. This may be due to smaller size of deep lymph nodes compared to superficial ones, which interfered appreciation of fat attenuation.

Similar to the literature, there was no significant difference between male and female with regard to number of superficial and deep lymph nodes [1]. However, mean maximum short axis diameter of lymph nodes in boys was statistically higher than in girls. The overall mean short axis diameters of the biggest lymph node for superficial and deep lymph nodes were noted as $7.55 \mathrm{~mm}$ and $4.79 \mathrm{~mm}$ in boys 
and $7.23 \mathrm{~mm}$ and $4.41 \mathrm{~mm}$ in girls. The differences between mean maximum short axis diameters of the biggest lymph nodes of boys and girls were calculated as $0.32 \mathrm{~mm}$ and $0.38 \mathrm{~mm}$ for superficial and deep lymph nodes respectively. Although statistically significant, these differences may be clinically insignificant.

\section{Limitations of the study}

There are several limitations to be considered. First, since it's ethically impossible to perform the CT examinations on a study group of healthy children due to well-known harmful effects of radiation exposure, we retrospectively evaluated previously acquired $\mathrm{CT}$ examinations of paediatric patients suffered from trauma. Second, the medical records of some patients were limited to trauma incident and consisted of no history of other diseases. So that, to accept all study patients as otherwise healthy may be inaccurate. Third, we did not have any histopathological proof verifying normality of lymph nodes. Lastly, as mentioned above, the number of girls recruited in the study, particularly in the age group of 10-11, was relatively less compared to boys.

\section{CONCLUSIONS}

Inguinal lymph nodes were multiple, symmetrical in size and number, commonly contained fat, and oval in shape in children. There was statistically significant difference between superficial and deep lymph nodes with regard to number, size and fat attenuation. Statistical difference was also detected between male and female in terms of short axis diameter in favour of male. The data determined about inguinal lymph node size in different paediatric age groups may be applicable as normative data in daily clinical CT evaluation practice.

\section{REFERENCES}

1. Bontumasi N, Jacobson JA, Caoili E, et al. Inguinal lymph nodes: size, number, and other characteristics in asymptomatic patients by CT. Surg Radiol Anat. 2014; 36(10): 1051-1055, doi: 10.1007/s00276-014-1255-0, indexed in Pubmed: 24435023.

2. de Carvalho JP, Patrício BF, Medeiros J, et al. Anatomic aspects of inguinal lymph nodes applied to lymphadenectomy in penile cancer. Adv Urol. 2011; 2011: 952532, doi: 10.1155/2011/952532, indexed in Pubmed: 22110493.

3. Esen G. Ultrasound of superficial lymph nodes. Eur J Radiol. 2006; 58(3): 345-359, doi: 10.1016/j.ejrad.2005.12.039, indexed in Pubmed: 16480846.

4. Grey AC, Carrington BM, Hulse PA, et al. Magnetic resonance appearance of normal inguinal nodes. Clin Radiol. 2000; 55(2): 124-130, doi: 10.1053/crad.1999.0330, indexed in Pubmed: 10657158.

5. McMahon CJ, Rofsky NM, Pedrosa I. Lymphatic metastases from pelvic tumors: anatomic classification, characterization, and staging. Radiology. 2010; 254(1): 31-46, doi: 10.1148/radiol.2541090361, indexed in Pubmed: 20032141

6. Morland B. Lymphadenopathy. Arch Dis Child. 1995; 73(5): 476-479, doi: 10.1136/adc.73.5.476, indexed in Pubmed: 8554372.

7. Oguz A, Karadeniz C, Temel EA, et al. Evaluation of peripheral lymphadenopathy in children. Pediatr Hematol Oncol. 2006; 23(7): 549-561, doi: 10.1080/08880010600856907, indexed in Pubmed: 16928650.

8. Paño B, Sebastià $C$, Buñesch $L$, et al. Pathways of lymphatic spread in male urogenital pelvic malignancies. Radiographics. 2011; 31(1): 135-160, doi: 10.1148/ rg.311105072, indexed in Pubmed: 21257939.

9. Ramirez $M$, Ingrand $P$, Richer JP, et al. What is the pelvic lymph node normal size? Determination from normal MRI examinations. Surg Radiol Anat. 2016; 38(4): 425-431, doi: 10.1007/s00276-015-1581-x, indexed in Pubmed: 26514962.

10. Roberto S, Valeria B, Roberto DV, et al. Analysis by high resolution ultrasound of superficial lymph nodes: anatomical, morphological and structural variations. Clin Imaging. 2014; 38(2): 96-99, doi: 10.1016/j.clinimag.2013.11.012, indexed in Pubmed: 24369877. 